\title{
铱催化串联不对称烯丙基取代/双键异构化构建轴手性化合物
}

\author{
赵庆如 $a, b \quad$ 蒋茹 $a$ 游书力*,a,b \\ ( ${ }^{a}$ 中国科学院上海有机化学研究所 金属有机化学国家重点实验室 上海 200032) \\ $\left({ }^{b}\right.$ 上海科技大学 物质科学与技术学院 上海 201210)
}

\begin{abstract}
摘要 轴手性化合物是一类重要的手性化合物, 其中苯乙烯类轴手性化合物因其轴手性稳定性相对较差, 目前高效不 对称合成的方法比较局限. 本工作以 $\beta$-萗酚作为亲核试剂, 通过将金属铱催化不对称烯丙基取代与双键异构化串联, 实现了中心手性到轴手性的转移, 从而高效地合成了一系列 $\beta$-萗酚衍生的苯乙烯类轴手性化合物.
\end{abstract}

关键词＼cjkstart轴手性; 不对称催化; 铱催化; 烯丙基取代; 烯烃异构化

\section{Ir-catalyzed Sequential Asymmetric Allylic Substitution/Olefin Isomerization for the Synthesis of Axially Chiral Compounds}

\author{
Qing-Ru Zhao $^{a, b} \quad$ Ru Jiang $^{a} \quad$ Shu-Li You*,a,b \\ ( ${ }^{a}$ State Key Laboratory of Organometallic Chemistry, Shanghai Institute of Organic Chemistry, \\ Chinese Academy of Sciences, Shanghai 200032, China) \\ ( ${ }^{b}$ School of Physical Science and Technology, ShanghaiTech University, Shanghai 201210, China)
}

\begin{abstract}
Axially chiral compounds represent an important class of chiral molecules. In this regard, many methods have been developed to access these compounds. However, efficient methods for the synthesis of axially chiral styrenes are limited to date, mainly due to their relative instability compared to axially chiral biaryl compounds. Iridium-catalyzed asymmetric allylic substitutions have evolved as a powerful tool in constructing $\mathrm{C}-\mathrm{C}$ or $\mathrm{C}-\mathrm{X}$ bonds at the allylic position. We developed an efficient sequential strategy to access a series of axially chiral styrenes by iridium-catalyzed allylic substitution and central-to-axial chirality transfer via olefin isomerization. With the iridium complex derived from $[\operatorname{Ir}(\operatorname{cod}) \mathrm{Cl}]_{2}$ and Alexakis ligand $\mathbf{L} 1$ as catalyst, and $\beta$-naphthol as nucleophiles, a broad range of axially chiral styrenes were obtained with moderate to excellent yields $(28 \% \sim 97 \%$ yields) and enantioselectivity $(59 \% \sim 98 \% e e)$. A general procedure for the asymmetric allylation of $\beta$-naphthol is described as the following: A flame-dried Schlenk tube was cooled to room temperature and filled with argon. To this flask were added $[\operatorname{Ir}(\operatorname{cod}) \mathrm{Cl}]_{2}(2.6 \mathrm{mg}, 0.004 \mathrm{mmol}, 2 \mathrm{~mol} \%),\left(S, S, S_{\mathrm{a}}\right)-\mathbf{L 1}(4.8 \mathrm{mg}, 0.008 \mathrm{mmol}, 4 \mathrm{~mol} \%)$, freshly distilled tetrahydrofuran (THF, $0.5 \mathrm{~mL})$ and propylamine $(0.5 \mathrm{~mL})$. The mixture was stirred at $50{ }^{\circ} \mathrm{C}$ for $30 \mathrm{~min}$ and then the low-boiling solvents were removed in vacuo to give a pale yellow solid. The solid was stirred at $50{ }^{\circ} \mathrm{C}$ again under vacuum until it became a powder. After that, $\beta$-naphthol 1 ( $0.22 \mathrm{mmol}, 1.1$ equiv.), allyl carbonate 2 ( $0.2 \mathrm{mmol}, 1.0$ equiv.), 1,4-diazobicyclo(2.2.2)octane (DABCO) $\left(67.3 \mathrm{mg}, 0.6 \mathrm{mmol}, 3.0\right.$ equiv.) and freshly distilled $\mathrm{Et}_{2} \mathrm{O}(2.0 \mathrm{~mL})$ were added to this flask under argon atmosphere. The reaction mixture was stirred at $20{ }^{\circ} \mathrm{C}$ until the starting material was consumed (monitored by thin layer chromatography, TLC). The crude reaction mixture was diluted with water $(5 \mathrm{~mL})$, and extracted with dichloromethane $\left(\mathrm{DCM}, 5 \mathrm{~mL} \times 3\right.$ ). The organic layers were collected, dried over $\mathrm{Na}_{2} \mathrm{SO}_{4}$ and then concentrated in vacuo to afford the crude product. The residue was purified by preparative TLC to afford the product.

Keywords axial chirality; asymmetric catalysis; iridium-catalyzed; allylic substitution; olefin isomerization
\end{abstract}

\section{1 引言}

轴手性化合物是一类因 $\sigma$ 键旋转受限而形成的手性 分子 ${ }^{[1]}$. 该类化合物因其独特的结构和性质广泛存在于 天然产物、生物活性分子以及材料分子中 ${ }^{[2]}$ (图 1a). 1980 年, 联芳基骨架的轴手性化合物 1, '- - -联荥-2,2'-双二苯膦 (BINAP) 作为手性配体被应用于不对称氢化反应中 ${ }^{[3]}$. 自此, 多种多样的轴手性化合物作为手性配体或催化剂
被广泛应用到不对称催化反应中 ${ }^{[4]}$. 其中, 联芳基类轴 手性化合物较为稳定且发展较为成熟[5], 而苯乙烯类轴 手性化合物, 由于其烯基和芳基之间的 $\sigma$ 键旋转能垒较 低, 轴手性相对不稳定. 因此, 高效立体选择性构建苯 乙烯类轴手性化合物一直是不对称合成领域的挑战 ${ }^{[6]}$ (图 1b).

在目前的报道中, 加成反应 ${ }^{[7]}$ 、偶联反应 ${ }^{[8]}$ 以及 $\mathrm{C}-$ $\mathrm{H}$ 键活化 ${ }^{[9]}$ 策略主要被用于催化不对称合成苯乙烯类型

\footnotetext{
*E-mail: slyou@sioc.ac.cn

Received July 11, 2021; published August 17, 2021.

Supporting information for this article is available free of charge via the Internet at http://sioc-journal.cn. Shanghai Municipality (No. 19590750400).

项目受国家自然科学基金(Nos. 21821002, 21961132002)和上海市科学技术委员会(No. 19590750400)资助.
}

Project supported by the National Natural Science Foundation of China (Nos. 21821002, 21961132002), and Science and Technology Commission of 


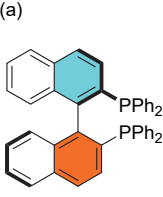

$(R)$-BINAP

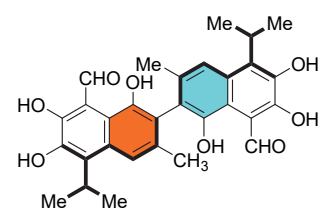

(R)-Gossypol
(S)-Marinopyrrole

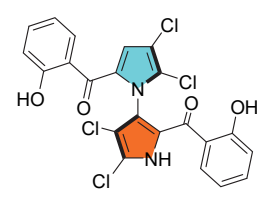

(b)

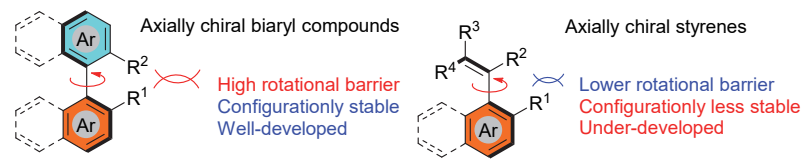

图 1 (a) 几种不同的轴手性化合物; (b)构建苯乙烯类型轴手性化合物 存在的挑战

Figure 1 (a) Selected examples of axially chiral compounds; (b) challenges existed in the synthesis of axially chiral styrenes

的轴手性化合物. 例如, 2016 年顾振华课题组 ${ }^{[10]}$ 通过金 属钯催化不对称偶联反应构建了一系列苯乙烯类轴手 性化合物(图 2a). 该反应可以以高达 99\%的收率和 $97 \%$ $e e$ 的对映选择性控制生成目标化合物. 除了以上三种策 略以外, 过渡金属催化串联不对称烯丙基取代与烯烃异 构化策略最近也被报道. 2020 年何英课题组 ${ }^{[11]}$ 利用金属 铱催化不对称烯丙基取代和 [1,3]-H 迁移串联反应, 高 效地实现了中心手性向轴手性的转移(图 2b). 该工作初 步揭示了金属铱催化的烯丙基取代反应在轴手性化合 物的构建方面的潜在应用价值.

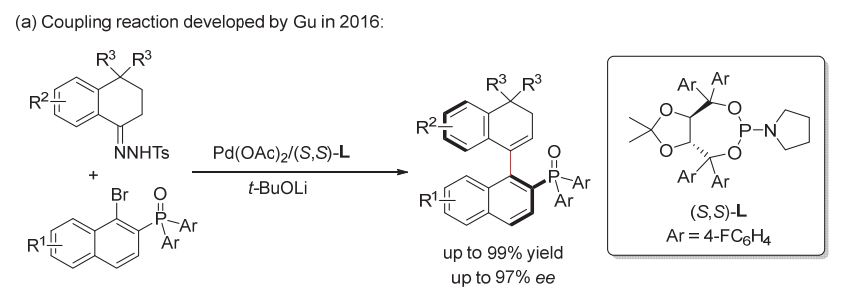

(b) Sequential reaction developed by He in 2020

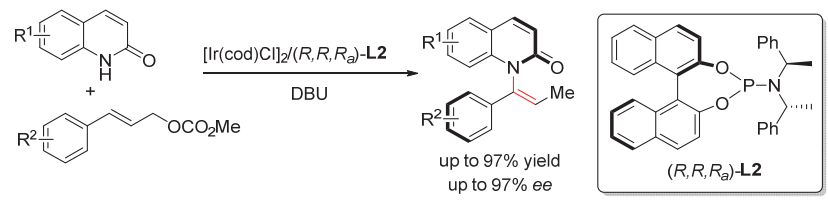

(c) This work

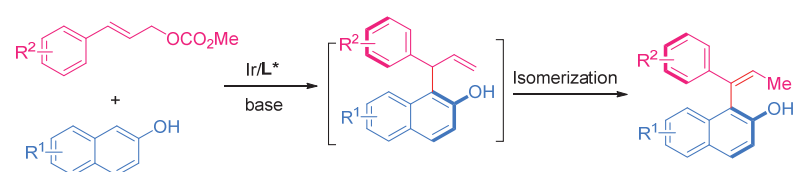

图 2 构建轴手性苯乙烯类化合物的不同策略

Figure 2 Various strategies for the synthesis of axially chiral styrenes

金属铱催化的不对称烯丙基取代反应作为构建 $\mathrm{C}-$ $\mathrm{C}$ 键或者 $\mathrm{C}-\mathrm{X}\left(\mathrm{X}=\mathrm{S}, \mathrm{O}, \mathrm{N}\right.$ 等)键的重要方法之一 ${ }^{[12-14]}$, 近年来已被广泛地用于实现多种富电子芳香化合物, 如

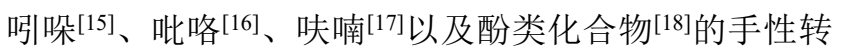
化. 据此, 我们设想富电子芳香化合物 $\beta$-䒬酚经铱催化 烯丙基取代反应生成的手性产物也可能通过 [1,3]-H 迁
移发生异构化, 实现中心手性向轴手性的转移(图 2c). 利用此策略可以实现苯乙烯类轴手性化合物的高效合 成 ${ }^{[19]}$.

\section{2 结果与讨论}

\section{1 反应条件篮选}

在初步尝试中, 我们以摩尔分数 $2 \%$ 的 $[\operatorname{Ir}(\operatorname{cod}) \mathrm{Cl}]_{2}$ 与摩尔分数 $4 \%$ 的 $\left(S, S, S_{a}\right)$-Alexakis 配体(L1) 原位制备的 铱配合物为催化剂, $\beta$-薺酚 $1 \mathrm{a}$ 与肉桂碳酸甲酯 $2 \mathrm{a}$ 为模

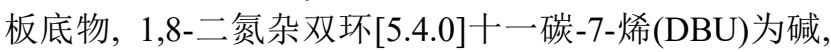
四氢呋喃(THF)为溶剂, $25{ }^{\circ} \mathrm{C}$ 下反应 $36 \mathrm{~h}$, 可以 $68 \%$ 的 核磁收率和 77\% ee 的对映选择性控制得到具有轴手性 的目标产物 4aa (表 1, Entry 1). 值得一提的是, 反应过 程中可以明显观察到烯丙基取代的中间体 3aa 的生成与 转化, 该现象表明反应依次经历了烯丙基取代和烯烃异 构化的历程.

在初步结果的基础上，我们对反应条件进行了优化 (表 1). 考虑到反应体系中的碱很可能对烯烃的异构化 过程影响较大, 我们首先分别对有机碱和无机碱进行了 考察(Entries 1 5). 从结果可以看出, 不同的碱对反应 的收率和对映选择性控制均有明显影响, 其中以 1,4-二 氮杂二环 [2.2.2]辛烷(DABCO) 为碱时, 反应效果最优, 可以 $90 \%$ 的核磁收率和 $87 \%$ ee 的对映选择性控制得到 目标产物 4aa (Entry 2). 因此, 接下来以 DABCO 为最优 碱对反应的溶剂进行考察(Entries 2，6 10). 当使用醚 类溶剂(Entries 2，6，7)或甲苯(Entry 9)时，反应效果较 好. 其中以 $\mathrm{Et}_{2} \mathrm{O}$ 为溶剂时, 可以将反应的对映选择性控 制提高至 $93 \%$ ee (Entry 7). 接着我们对配体也进行了考 察(Entries 7, 11 14). 对于 $\left(S, S, S_{\mathrm{a}}\right)$ 构型的 Feringa 类型的 亚磷酰胺配体 L1 (Alexakis 配体) ${ }^{[20]}$ 和 L2 (Feringa 配 体 $)^{[21]}$, 反应效果均较好, 可以取得基本相当的结果 (Entry 7, 91\% yield, 93\% ee; Entry 11, 96\% yield, 90\% $e e)$. 而对于 Me-THQphos $\left(S, S_{a}\right)$-L4 和 BHPphos $\left(S_{a}\right)-\mathbf{L} \mathbf{5}$, 反应的活性明显下降(Entry 13, 12\% yield, 55\% ee; Entry 14: trace). 此外, 我们还尝试了 $\left(S, S, R_{\mathrm{a}}\right)$ 构型的 Feringa 配 体 $\mathbf{L 3}$, 反应基本无法发生，这可能是因为该类配体形成 的铱络合物较难发生 $\mathrm{C}-\mathrm{H}$ 键活化, 从而难以形成具有 催化活性的一价铱物种 (Entry 12) ${ }^{[22]}$. 鉴于使用 $\left(S, S, S_{a}\right)$ - $\mathbf{L} \mathbf{1}$ 时, 反应的对映选择性控制较好, 因此选用 $\left(S, S, S_{a}\right)-\mathbf{L} \mathbf{1}$ 作为反应的最优配体. 考虑到产物的轴手性 不稳定，易消旋，我们对反应温度也进行了考察. 降温 至 $20{ }^{\circ} \mathrm{C}$ 时(Entry 15), 虽然反应的对映选择性控制仅有 微小提升 $(94 \% e e)$, 但核磁收率可以提升至 $97 \%$ yield(分离收率 $93 \%$ yield). 而进一步降温至 $10{ }^{\circ} \mathrm{C}$ 时, 反应核磁收率明显降低(Entry 16, 82\% yield, 95\% ee). 总体而言, $20{ }^{\circ} \mathrm{C}$ 时，反应效果最优，可以 $97 \%$ 的核磁收 率和 $94 \%$ ee 的对映选择性控制得到目标产物 4aa (Entry 15), 因此选择 $20{ }^{\circ} \mathrm{C}$ 为最优温度. 最后, 我们对碱的用 
量进行了考察, 随着碱的用量减少, 反应收率与对映选 择性控制均下降(Entries 15，17，18), 例如，使用摩尔分 数 $50 \% \mathrm{DABCO}$ 进行反应，反应仅能以 $72 \%$ 的核磁收率 和 $84 \%$ ee 的对映选择性控制得到产物(Entry 17). 因此, 选择 3.0 equiv. DABCO 作为碱的最优用量. 综上所述, 通过一系列条件优化, 我们确定了反应的最优条件, 即 以摩尔分数 $2 \%$ 的 $[\operatorname{Ir}(\operatorname{cod}) \mathrm{Cl}]_{2}$ 与摩尔分数 $4 \%$ 的 $\left(S, S, S_{a}\right)-$ L1 原位制备形成的铱络合物为催化剂, 3.0 equiv. $\mathrm{DABCO}$ 为碱, $\mathrm{Et}_{2} \mathrm{O}$ 为溶剂, 1.1 equiv. 1 与 1.0 equiv. 2 , 在 $20{ }^{\circ} \mathrm{C}$ 下进行反应(详细的条件考察以及中间体 $\mathbf{3 a a}$ 的 转化与手性数据变化情况, 请参考 Supporting Information 的 $\mathrm{S} 3 \sim \mathrm{S} 4$ 页 Table $\mathrm{S} 1$ ).

\section{表 1 反应条件优化 ${ }^{a}$}

Table 1 Optimization of reaction conditions

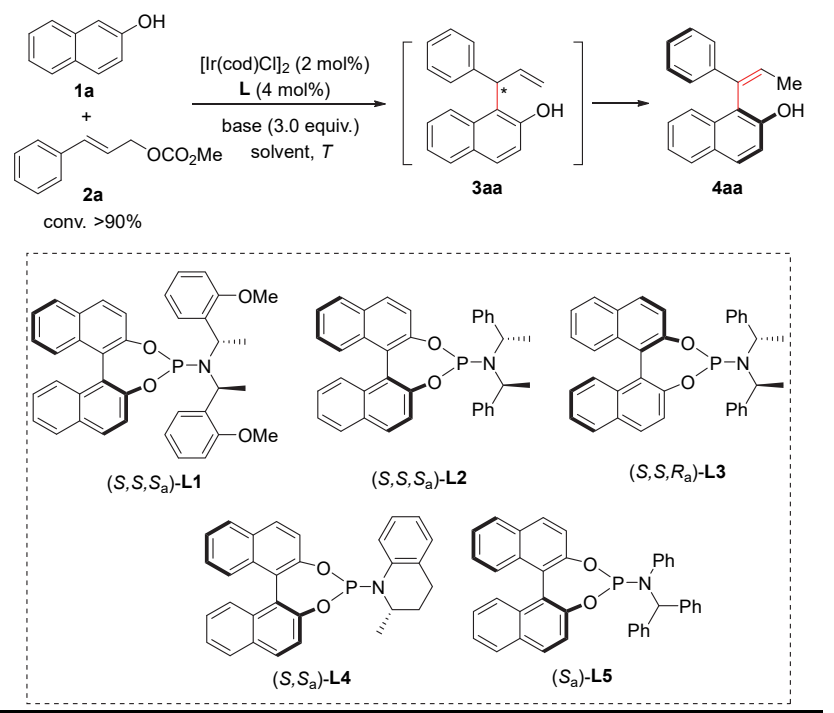

\begin{tabular}{|c|c|c|c|c|c|c|}
\hline \multirow{2}{*}{ Entry } & \multirow{2}{*}{ Base } & \multirow{2}{*}{ Solvent } & \multirow{2}{*}{$\mathbf{L}$} & \multirow{2}{*}{$T /{ }^{\circ} \mathrm{C}$} & \multicolumn{2}{|c|}{ 4aa } \\
\hline & & & & & Yield $^{b}$ & $e e^{c}$ \\
\hline 1 & DBU & THF & L1 & 25 & $68 \%$ & $77 \%$ \\
\hline 2 & DABCO & THF & L1 & 25 & $90 \%$ & $87 \%$ \\
\hline 3 & TBD & THF & L1 & 25 & $17 \%$ & $49 \%$ \\
\hline 4 & $\mathrm{Cs}_{2} \mathrm{CO}_{3}$ & THF & L1 & 25 & $83 \%$ & $43 \%$ \\
\hline 5 & $t$-BuONa & THF & L1 & 25 & $48 \%$ & $83 \%$ \\
\hline 6 & DABCO & 1,4-dioxane & L1 & 25 & $59 \%$ & $71 \%$ \\
\hline 7 & DABCO & $\mathrm{Et}_{2} \mathrm{O}$ & L1 & 25 & $91 \%$ & $93 \%$ \\
\hline 8 & DABCO & DCM & L1 & 25 & $36 \%$ & $74 \%$ \\
\hline 9 & DABCO & toluene & L1 & 25 & $98 \%$ & $72 \%$ \\
\hline 10 & $\mathrm{DABCO}$ & $\mathrm{MeCN}$ & L1 & 25 & $38 \%$ & $55 \%$ \\
\hline 11 & DABCO & $\mathrm{Et}_{2} \mathrm{O}$ & $\mathbf{L 2}$ & 25 & $96 \%$ & $90 \%$ \\
\hline 12 & DABCO & $\mathrm{Et}_{2} \mathrm{O}$ & $\mathbf{L 3}$ & 25 & trace & N.D. ${ }^{d}$ \\
\hline 13 & $\mathrm{DABCO}$ & $\mathrm{Et}_{2} \mathrm{O}$ & L4 & 25 & $12 \%$ & $55 \%$ \\
\hline 14 & DABCO & $\mathrm{Et}_{2} \mathrm{O}$ & $\mathbf{L 5}$ & 25 & trace & N.D. ${ }^{d}$ \\
\hline 15 & DABCO & $\mathbf{E t}_{2} \mathbf{O}$ & $\mathbf{L 1}$ & 20 & $\begin{array}{c}97 \% \\
(93 \%)\end{array}$ & $94 \%$ \\
\hline 16 & DABCO & $\mathrm{Et}_{2} \mathrm{O}$ & L1 & 10 & $82 \%$ & $95 \%$ \\
\hline $17^{f}$ & DABCO & $\mathrm{Et}_{2} \mathrm{O}$ & L1 & 20 & $84 \%$ & $90 \%$ \\
\hline $18^{g}$ & DABCO & $\mathrm{Et}_{2} \mathrm{O}$ & L1 & 20 & $72 \%$ & $84 \%$ \\
\hline
\end{tabular}

${ }^{a}$ Reaction conditions: 1a $(0.22 \mathrm{mmol}), \mathbf{2 a}(0.2 \mathrm{mmol}),[\mathrm{Ir}(\operatorname{cod}) \mathrm{Cl}]_{2}(0.004$ $\mathrm{mmol})$, L1 $(0.008 \mathrm{mmol})$, base $(0.6 \mathrm{mmol})$, solvent $(2.0 \mathrm{~mL}) .{ }^{b}$ Determined by ${ }^{1} \mathrm{H}$ NMR analysis of the crude product with $\mathrm{CH}_{2} \mathrm{Br}_{2}$ as an internal standard. ${ }^{c}$ Determined by HPLC analysis. ${ }^{d}$ N.D.: not detected. ${ }^{e}$ Isolated yield. ${ }^{f}$ Using 1.0 equiv. DABCO. ${ }^{g}$ Using 50 mol\% DABCO.

\section{2 底物拓展}

在确定了反应的最优条件后(表 1 , Entry 15), 我们 对该串联反应的底物普适性进行了考察.

首先以 $\beta$-菜酚 $1 \mathrm{a}$ 为底物, 对不同取代的肉桂碳酸 甲酯的普适性进行了研究(表 2). 当苯环对位或者间位 带有给电子取代基，如甲基( $\mathrm{Me}, \mathbf{2 b}, \mathbf{2 h})$ 、甲氧基 $(\mathrm{OMe}$, 2c, 2i) 以及异丁基 $(i-\mathrm{Bu}, \mathbf{2 d})$ 时，反应均可以较好兼容, 取得良好至优秀的收率以及对映选择性控制( $\mathbf{4 a b} \sim \mathbf{4 a d}$, 4ah, 4ai, $77 \% \sim 93 \%$ yields, $80 \% \sim 94 \% e e$ ). 相比于给电 子取代基，当苯环的对位带有卤原子，如氟 $(\mathrm{F}, 2 \mathrm{ff}) 、$ 溴 $(\mathrm{Br}, \mathbf{2 g})$, 虽然反应的收率略有下降，但仍然可以取得优 秀的对映选择性控制 (4af, 72\% yield, $85 \% e e ; \mathbf{4 a g}, 76 \%$ yield, $93 \% e e$ ). 当苯环间位连有卤原子时, 反应能够以 优秀的收率生成目标化合物, 但仅能取得中等至良好的 对映选择性控制(4aj～4al，92\% 94\% yields，73\% $85 \% e e$ ). 值得一提的是, 对于其他芳环衍生的肉桂碳酸 酯底物, 如 1,2-亚甲二氧基苯、蒜, 也可以很好地兼容 于这一反应体系(4am, 58\% yield, 92\% ee；4an, 94\% yield, $84 \% e e$ ). 当苯环邻位带有取代基时, 如甲基( $\mathrm{Me}$,

表 2 烯丙基碳酸酯底物反应普适性研究 ${ }^{a}$

Table 2 Substrate scope for allyl carbonates

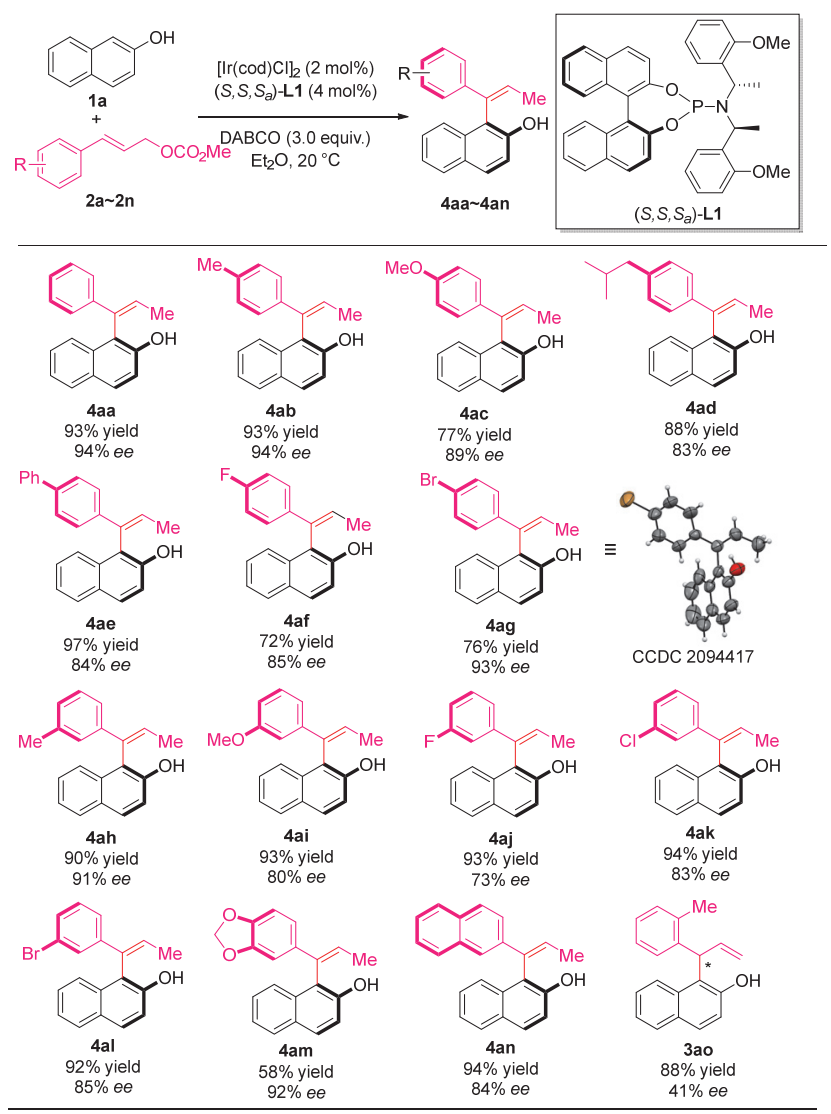

a Reaction conditions: 1a $(0.22 \mathrm{mmol}), 2(0.2 \mathrm{mmol}),[\operatorname{Ir}(\operatorname{cod}) \mathrm{Cl}]_{2}(0.004$ $\mathrm{mmol}),\left(S, S, S_{a}\right)-\mathbf{L 1}(0.008 \mathrm{mmol}), \mathrm{DABCO}(0.6 \mathrm{mmol})$, and $\mathrm{Et}_{2} \mathrm{O}(2.0 \mathrm{~mL})$ at $20{ }^{\circ} \mathrm{C}$. Isolated yield. The $e e$ values of products were determined by HPLC analysis (see the SI for details). 
20), 反应仅能获得中间体 3 ao ( $88 \%$ yield, $41 \%$ ee), 这 可能是由于苯环邻位带有取代基时，中间体 $\mathbf{3}$ 位阻较大, 不利于双键异构化. 此外, 我们培养了对映纯产物 $\mathbf{4 a g}$ 的单晶, 并通过 X 射线衍射, 确定了 $4 \mathrm{ag}$ 的结构以及绝 对构型 $\left(S_{a}\right)$, 其它产物的绝对构型据此推定(详见 $\mathrm{SI}$ ).

接下来以肉桂碳酸甲酯 $2 \mathbf{a}$ 为底物, 对 $\beta$-萗酚衍生 物的普适性进行了考察(表 3). 当䒺酚的 C6 位带有乙基 $(\mathrm{Et}, \mathbf{1 b})$ 或者溴原子 $(\mathrm{Br}, 1 \mathrm{~d})$ 时, 反应能够以中等的收率 以及良好的对映选择性控制得到目标产物 (4ba, $61 \%$ yield, 85\% ee; 4da, 51\% yield, 70\%ee). 值得注意的是, 菜环的不同位点带有甲氧基取代基时, 反应结果具有明 显差异，如甲氧基位于 C6 位时，反应虽然可以取得良 好的对映选择性控制，但收率明显降低(4ca, 28\% yield, $86 \% \mathrm{ee}$ ), 这可能是因为该位点上甲氧基的强给电子效 应明显增强了菜酚 $\mathrm{C} 2$ 位上酚羟基的亲核性, 从而导致 体系中有醚化副产物的生成. 而䒺酚的 C7 位上的甲氧 基取代基对 $\mathrm{C} 2$ 位上的酚羟基亲核性无明显影响, 该反 应能够以中等的收率以及对映选择性控制得到目标产 物(4ea, 70\% yield, 74\% ee). 当萗酚的 C8 位带有溴原子, 目标产物 $\mathbf{4 f a}$ 的消旋化能垒明显上升, 反应可以取得优 秀的对映选择性控制(4fa, 45\% yield, $98 \%$ ee). 对于菲衍 生的亲核试剂, 反应也可以取得中等的结果(4ga, 49\% yield, 59\% ee).

\section{表 $3 \beta$-萗酚底物反应普适性研究 ${ }^{a}$}

Table 3 Substrate scope for $\beta$-naphthols
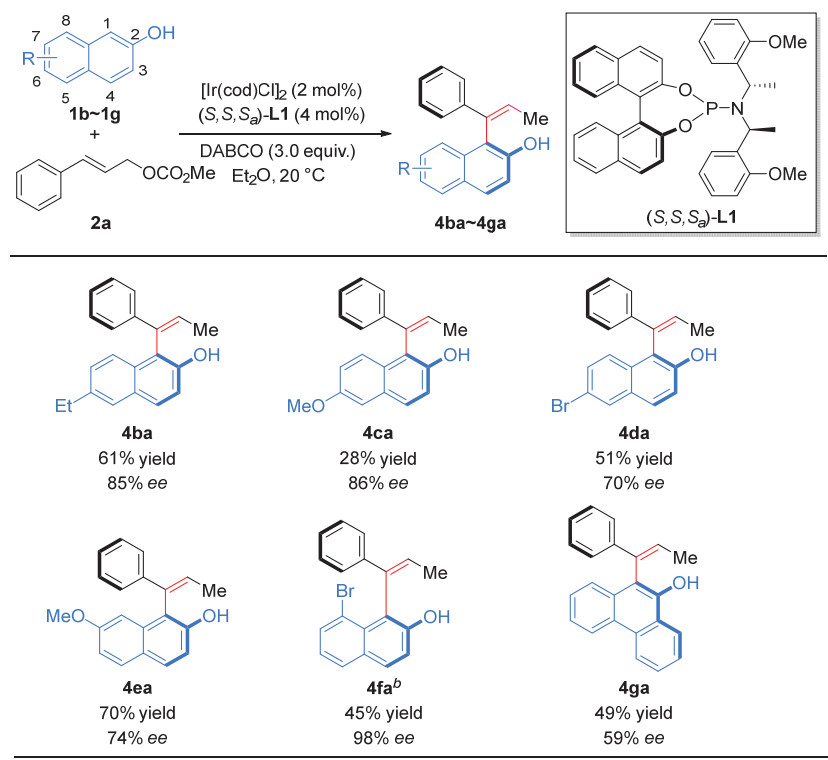

${ }^{a}$ Reaction conditions: 1 (0.22 mmol), 2a $(0.2 \mathrm{mmol}),[\operatorname{Ir}(\operatorname{cod}) \mathrm{Cl}]_{2}(0.004$ $\mathrm{mmol}),\left(S, S, S_{a}\right)-\mathbf{L 1}(0.008 \mathrm{mmol}), \mathrm{DABCO}(0.6 \mathrm{mmol})$, and $\mathrm{Et}_{2} \mathrm{O}(2.0 \mathrm{~mL})$ at $20{ }^{\circ} \mathrm{C}$. Isolated yield. The ee values of products were determined by HPLC analysis (see the SI for details). ${ }^{b} \mathrm{DBU}(0.6 \mathrm{mmol})$ was used as base.

\section{3 反应机理研究}

\subsection{1 控制实验}

如上所述, 反应过程中可以观察到烯丙基取代的中 间体 $3 \mathbf{a a}$ 的生成与转化, 该现象表明反应大致经历了两
个历程: (1)铱催化烯丙基取代反应，即中心手性的构建; (2)烯烃异构化反应，即中心手性向轴手性的转移. 这两 个过程均涉及手性中心的变化, 因此均可能明显影响反 应的对映选择性控制. 鉴于 Feringa 类型配体衍生的铱 催化剂在烯丙基取代反应中能以较好的对映选择性控

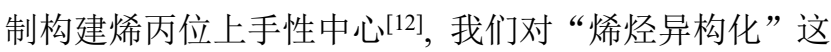
一历程的影响因素进行了研究.

通过表 4 可以看出, 在标准条件下, 中间体 $\mathbf{3 a a}$ 可 以顺利转化为目标化合物(Entry 1, 68\% yield), 其对映 选择性基本可以得到保持(3aa, 80\% ee; 4aa, 77\% ee), 而仅在碱或者铱催化剂的促进下，中间体 $\mathbf{3 a a}$ 转化率较 低, 有微量的目标化合物生成(Entry 2,8\% yield; Entry 3, 7\% yield)且对映选择性均有明显下降(Entry 2, 3aa, 94\% ee; 4aa, 80\% ee; Entry 3, 3aa, 80\% ee; 4aa, 71\% ee). 以上控制实验结果表明中间体 $3 \mathbf{a a}$ 向产物 $4 \mathbf{a a}$ 转化需要 铱催化剂与碱的共同参与.

表 4 控制实验

Table 4 Control experiment

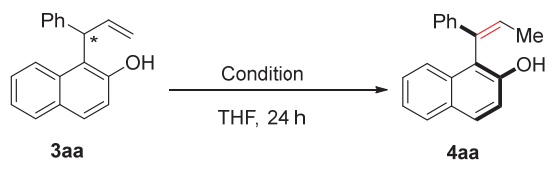

\begin{tabular}{ccccc}
\hline Entry & Condition & $\begin{array}{c}\text { NMR yield } \\
\text { of 4aa }\end{array}$ & $\begin{array}{c}\text { ee of } \\
\text { 3aa }\end{array}$ & $\begin{array}{c}\text { ee of } \\
\text { 4aa }\end{array}$ \\
\hline \multirow{2}{*}{1} & $\begin{array}{c}{[\operatorname{Ir}(\operatorname{cod}) \mathrm{Cl}]_{2}(2 \mathrm{~mol} \%)} \\
\left(S, S, S_{a}\right)-\mathbf{L 1}(4 \mathrm{~mol} \%)\end{array}$ & $68 \%$ & $80 \%$ & $77 \%$ \\
& DBU (3.0 equiv. $)$ & & & \\
2 & Without DBU & $8 \%$ & $94 \%$ & $80 \%$ \\
3 & Without $[\mathrm{Ir}]$ catalyst & $7 \%$ & $80 \%$ & $71 \%$ \\
\hline
\end{tabular}

\subsection{2 反应可能的机理}

根据控制实验的结果, 我们提出了如图 3 所示催化 循环，首先一价铱络合物与烯丙基底物 $\mathbf{2 a}$ 进行配位，发 生氧化加成生成 $\pi$-烯丙基铱物种 II; 随后该物种接受亲

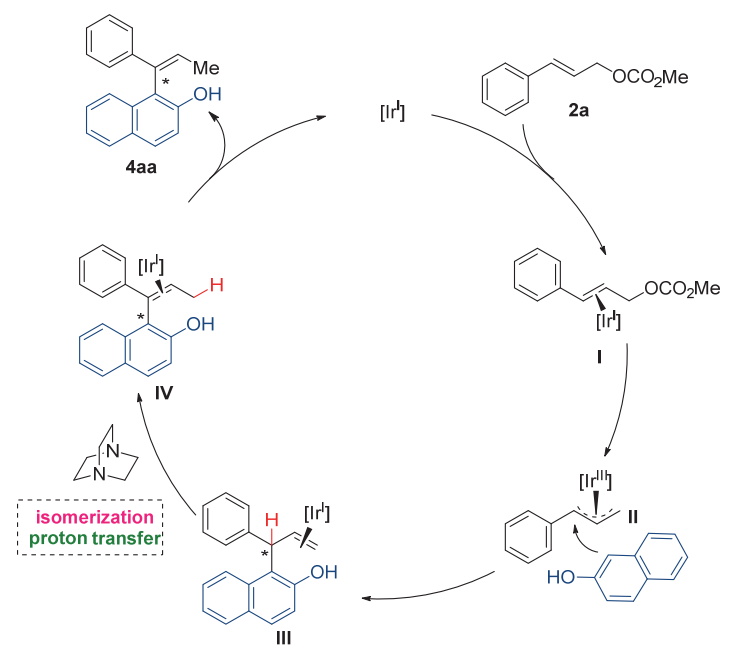

图 3 可能的催化循环

Figure 3 Proposed catalytic circle 
核试剂进攻生成中间体 3aa 配位的铱物种 III; 再在碱 和金属铱催化剂的共同作用下, 中间体 $\mathbf{3 a a}$ 烯丙位上的 氢发生 [1,3]-H 迁移, 实现中心手性向轴手性的转移 ${ }^{[19]}$; 最后金属铱催化剂解离, 得到目标产物 $4 \mathbf{a a}$, 完成催化 循环.

\section{3 结论}

本工作利用金属铱催化分子间串联不对称烯丙基 取代/烯烃异构化反应, 实现了中心手性向轴手性的转 变, 高效地构建了一系列由 $\beta$-䒺酚衍生的轴手性苯乙烯 类化合物. 该方法具有良好的底物普适性, 为轴手性苯 乙烯类化合物的构建提供了一种有效的合成方法.

\section{References}

[1] For reviews: (a) Kumarasamy, E.; Raghunathan, R.; Sibi, M. P.; Sivaguru, J. Chem. Rev. 2015, 115, 11239. (b) Wang, Y.-B.; Tan, B. Acc. Chem. Res. 2018, 51, 534. (c) Zhang, S.; Liao, G.; Shi, B. Chin. J. Org. Chem. 2019, 39, 1522 (in Chinese). (张硕, 廖港, 史炳锋, 有机化学, 2019, 39, 1522.) (d) Cheng, J. K.; Xiang, S.-H.; Li, S.; Ye, L.; Tan, B. Chem. Rev. 2021, 121, 4805.

[2] For reviews: (a) Kozlowski, M. C.; Morgan, B. J.; Linton, E. C. Chem. Soc. Rev. 2009, 38, 3193. (b) Bringmann, G.; Gulder, T.; Gulder, T. A.; Breuning, M. Chem. Rev. 2011, 111, 563. (c) Erbas-Cakmak, S.; Leigh, D. A.; McTernan, C. T.; Nussbaumer, A. L. Chem. Rev. 2015, 115, 10081 .

[3] Miyashita, A.; Yasuda, A.; Takaya, H.; Toriumi, K.; Ito, T.; Souchi, T.; Noyori, R. J. Am. Chem. Soc. 1980, 102, 7932.

[4] For books: (a) Zhou, Q.-L. Privileged Chiral Ligands and Catalysts, Wiley-VCH, Weinheim, Germany, 2011. (b) You, S.-L. Asymmetric Dearomatization Reactions, Wiley-VCH, Weinheim, Germany, 2016. For review: (c) Li, Y.-M.; Kwong, F.-Y.; Yu, W.-Y.; Chan, A. S. C. Coord. Chem. Rev. 2007, 251, 2119.

[5] For reviews: (a) Wencel-Delord, J.; Panossian, A.; Leroux, F. R.; Colobert, F. Chem. Soc. Rev. 2015, 44, 3418. (b) Loxq, P.; Manoury, E.; Poli, R.; Deydier, E.; Labande, A. Coord. Chem. Rev. 2016, 308, 131. (c) Wang, Q.; Gu, Q.; You, S.-L. Acta Chim. Sinica 2019, 77, 690 (in Chinese). (王强, 顾庆, 游书力, 化学学报, 2019, 77, 690.)

[6] Gu, Z.; Feng, J. SynOpen 2021, 5, 68.

[7] For selected examples: (a) Zheng, S.-C.; Wu, S.; Zhou, Q.; Chung, L. W.; Ye, L.; Tan, B. Nat. Commun. 2017, 8, 15238. (b) Tan, Y.; Jia, S.; Hu, F.; Liu, Y.; Peng, L.; Li, D.; Yan, H. J. Am. Chem. Soc. 2018, 140, 16893. (c) Jia, S.; Chen, Z.; Zhang, N.; Tan, Y.; Liu, Y.; Deng, J.; Yan, H. J. Am. Chem. Soc. 2018, 140, 7056. (d) Wang, C.-S.; Li, T.-Z.; Liu, S.-J.; Zhang, Y.-C.; Deng, S.; Jiao, Y.; Shi, F. Chin. J. Chem. 2020, 38, 543. (e) Sheng, F.-T.; Li, Z.-M.; Zhang, Y.-Z.; Sun, L.-X.; Zhang, Y.-C.; Tan, W.; Shi, F. Chin. J. Chem. 2020, 38, 583. (f) Wang, J.-Y.; Sun, M.; Yu, X.-Y.; Zhang, Y.-C.; Tan, W.; Shi, F. Chin. J. Chem. 2021, 39, 2163.

[8] For selected examples: (a) Bringmann, G.; Price Mortimer, A. J.; Keller, P. A.; Gresser, M. J.; Garner, J.; Breuning, M. Angew. Chem. Int. Ed. 2005, 44, 5384. (b) Pan, C.; Zhu, Z.; Zhang, M.; Gu, Z. Angew. Chem. Int. Ed. 2017, 56, 4777.

[9] For selected examples: (a) Wang, F.; Qi, Z.; Zhao, Y.; Zhai, S.; Zheng, G.; Mi, R.; Huang, Z.; Zhu, X.; He, X.; Li, X. Angew. Chem. Int. Ed. 2020, 59, 13288. (b) Yang, C.; Wu, T.-R.; Li, Y.; Wu, B.-B.; Jin, R.-X.; Hu, D.-D.; Li, Y.-B.; Bian, K.-J.; Wang, X.-S. Chem. Sci. 2021, 12, 3726.

[10] Feng, J.; Li, B.; He, Y.; Gu, Z. Angew. Chem. Int. Ed. 2016, 55, 2186.

[11] Sun, C.; Qi, X.; Min, X.-L.; Bai, X.-D.; Liu, P.; He, Y. Chem. Sci. 2020, 11, 10119.

[12] For reviews of allylic substitution reactions: (a) Zhang, M.-M.; Luo, Y.-Y.; Lu, L.-Q.; Xiao, W.-J. Acta Chim. Sinica 2018, 76, 838 (in Chinese). (张毛毛, 骆元元, 陆良秋, 肖文精, 化学学报, 2018, 76, 838.) (b) Ma, X.; Yu, J.; Wang, Z.; Zhang, Y.; Zhou, Q. Chin. J. Org. Chem. 2020, 40, 2669 (in Chinese). (马献涛, 于静, 王子龙, 张望, 周秋菊, 有机化学, 2020, 40, 2669.) For selected examples of allylic substitution reactions: (c) Yao, K.; Liu, H.; Yuan, Q.; Liu, Y.; Liu, D.; Zhang, W. Acta Chim. Sinica 2019, 77, 993 (in Chinese). (姚坤, 刘浩, 袁乾家, 刘燕刚, 刘德龙, 张万斌, 化学学报, 2019 , 77, 993.) (d) Xiao, J.; Xu, H.; Huo, X.; Zhang, W.; Ma, S. Chin. J. Chem. 2021, 39, 1958. (e) Huo, X.; Zhao, L.; Luo, Y.; Wu, Y.; Sun, Y.; Li, G.; Gridneva, T.; Zhang, J.; Ye, Y.; Zhang, W. CCS Chem. 2021, 3, 1933.

[13] For reviews of iridium-catalyzed allylic substitution reactions: (a) Hartwig, J. F.; Stanley, L. M. Acc. Chem. Res. 2010, 43, 1461. (b) Qu, J.; Helmchen, G. Acc. Chem. Res. 2017, 50, 2539. (c) Deng, Y.; Yang, W.; Yang, X.; Yang, D. Chin. J. Org. Chem. 2017, 37, 3039 (in Chinese). (邓颖颖，杨文，杨新，杨定乔，有机化学，2017，37, 3039.) (d) Cheng, Q.; Tu, H.-F.; Zheng, C.; Qu, J.-P.; Helmchen, G.; You, S.-L. Chem. Rev. 2019, 119, 1855. (e) Tian, F.; Zhang, J.; Yang, W.; Deng, W. Chin. J. Org. Chem. 2020, 40, 3262 (in Chinese). (田 飞, 张键, 杨武林, 邓卫平, 有机化学, 2020, 40, 3262.

[14] For selected examples of iridium-catalyzed allylic substitution reactions: (a) Krautwald, S.; Sarlah, D.; Schafroth, M. A.; Carreira, E. M. Science 2013, 340, 1065. (b) Liu, W.-B.; Reeves, C. M.; Stoltz, B. M. J. Am. Chem. Soc. 2013, 135, 17298. (c) Liu, J.; Cao, C.-G.; Sun, H.-B.; Zhang, X.; Niu, D. J. Am. Chem. Soc. 2016, 138, 13103. (d) Huo, X.; He, R.; Zhang, X.; Zhang, W. J. Am. Chem. Soc. 2016 138, 11093. (e) Huo, X.; Zhang, J.; Fu, J.; He, R.; Zhang, W. J. Am. Chem. Soc. 2018, 140, 2080. (f) Wei, L.; Zhu, Q.; Xu, S.-M.; Chang, X.; Wang, C.-J. J. Am. Chem. Soc. 2018, 140, 1508. (g) Xu, S.-M.; Wei, L.; Shen, C.; Xiao, L.; Tao, H.-Y.; Wang, C.-J. Nat. Commun. 2019, 10, 5553. (h) Han, M.; Yang, M.; Wu, R.; Li, Y.; Jia, T.; Gao, Y.; Ni, H.-L.; Hu, P.; Wang, B.-Q.; Cao, P. J. Am. Chem. Soc. 2020, 142, 13398. (i) Yang, P.; Liu, C.-X.; Zhang, W.-W.; You, S.-L. Acta Chim. Sinica. 2021, 79, 742 (in Chinese). (杨普苏, 刘晨旭, 张文 文, 游书力, 化学学报, 2021, 79, 742.)

[15] For selected examples: (a) Liu, W.-B.; He, H.; Dai, L.-X.; You, S.-L. Org. Lett. 2008, 10, 1815. (b) Wu, Q.-F.; He, H.; Liu, W.-B.; You, S.-L. J. Am. Chem. Soc. 2010, 132, 11418. (c) Huang, L.; Dai, L.-X.; You, S.-L. J. Am. Chem. Soc. 2016, 138, 5793. (d) Jiang, S.-Z.; Zeng, X.-Y.; Liang, X.; Lei, T.; Wei, K.; Yang, Y.-R. Angew. Chem. Int. Ed. 2016, 55, 4044. (e) Tu, H.-F.; Zhang, X.; Zheng, C.; Zhu, M.; You, S.-L. Nat. Catal. 2018, 1, 601. (f) Huang, L.; Cai, Y.; Zhang, H.-J.; Dai, L.-X.; You, S.-L. CCS Chem. 2019, 1, 106. (g) Uno, H.; Kawai, K.; Shiro, M.; Shibata, N. ACS Catal. 2020, 10, 14117. (h) Jiang, R.; Ding, L.; Zheng, C.; You, S.-L. Science 2021, 371, 380. (i) Zhang, J.; Gao, Y.-S.; Gu, B.-M.; Yang, W.-L.; Tian, B.-X.; Deng, W.-P. ACS Catal. 2021, 11, 3810.

[16] For selected examples: (a) Zhuo, C.-X.; Liu, W.-B.; Wu, Q.-F.; You, S.-L. Chem. Sci. 2012, 3, 205. (b) Zhuo, C.-X.; Wu, Q.-F; Zhao, Q.; Xu, Q.-L.; You, S.-L. J. Am. Chem. Soc. 2013, 135, 8169. (c) Zhuo, C.-X.; Cheng, Q.; Liu, W.-B.; Zhao, Q.; You, S.-L. Angew. Chem. Int. Ed. 2015, 54, 8475. (d) Huang, L.; Cai, Y.; Zheng, C.; Dai, L.-X.; You, S.-L. Angew. Chem. Int. Ed. 2017, 56, 10545. (e) Zi, Y.; Lange, M.; Schultz, C.; Vilotijevic, I. Angew. Chem. Int. Ed. 2019, $58,10727$.

[17] For selected examples: (a) Bechem, B.; Patman, R. L.; Hashmi, A. S. K.; Krische, M. J. J. Org. Chem. 2010, 75, 1795. (b) Chen, W.; Hartwig, J. F. J. Am. Chem. Soc. 2012, 134, 15249.

[18] For selected examples: (a) Nemoto, T.; Ishige, Y.; Yoshida, M.; Kohno, Y.; Kanematsu, M.; Hamada, Y. Org. Lett. 2010, 12, 5020. (b) Wu, Q.-F.; Liu, W.-B.; Zhuo, C.-X.; Rong, Z.-Q.; Ye, K.-Y.; You, S.-L. Angew. Chem., Int. Ed. 2011, 50, 4455. (c) Xu, Q.-L.; Dai, L.-X.; You, S.-L. Org. Lett. 2012, 14, 2579. (d) Zhuo, C.-X.; You, S.-L. Angew. Chem., Int. Ed. 2013, 52, 10056. (e) Cheng, Q.; Wang, Y.; You, S.-L. Angew. Chem., Int. Ed. 2016, 55, 3496. (f) Tu, H.-F.; Zheng, C.; Xu, R.-Q.; Liu, X.-J.; You, S.-L. Angew. Chem. Int. Ed. 2017, 56, 3237. (g) Shen, D.; Chen, Q.; Yan, P.; Zeng, X.; Zhong, G. Angew. Chem. Int. Ed., 2017, 56, 3242.

[19] Computational studies of the isomerization process were developed by He and coworkers: Wang, J.; Qi, X.; Min, X.-L.; Yi, W.; Liu, P.; He, Y. J. Am. Chem. Soc. 2021, 143, 10686.

[20] For selected examples: (a) Bartels, B.; García-Yebra, C.; Helmchen, G. Eur. J. Org. Chem. 2003, 1097. (b) Alexakis, A.; Polet, D. Org. Lett. 2004, 6, 3529. (c) Tissot-Croset, K.; Polet, D.; Alexakis, A. Angew. Chem. Int. Ed. 2004, 43, 2426. (d) Spiess, S.; Welter, C.; Franck, G.; Taquet, J.-P.; Helmchen, G. Angew. Chem. Int. Ed. 2008, 47, 7652. (e) Spiess, S.; Raskatov, J. A.; Gnamm, C.; Brodner, K.; Helmchen, G. Chem. Eur. J. 2009, 15, 11087. (f) Raskatov, J. A.; Spiess, S.; Gnamm, C.; Brodner, K.; Rominger, F.; Helmchen, G. Chem. Eur. J. 2010, 16, 6601. 
[21] For selected examples: (a) de Vries, A. H. M.; Meetsma, A.; Feringa, B. L. Angew. Chem. Int. Ed. 1996, 35, 2374. (b) Feringa, B. L.; Pineschi, M.; Arnold, L. A.; Imbos, R.; de Vries, A. H. M. Angew. Chem. Int. Ed. 1997, 36, 2620. (c) Ohmura, T.; Hartwig, J. F. J. Am. Chem. Soc. 2002, 124, 15164. (d) López, F.; Ohmura, T.; Hartwig, J. F. J. Am. Chem. Soc. 2003, 125, 3426. (e) Kiener, C. A.; Shu, C.;
Incarvito, C.; Hartwig, J. F. J. Am. Chem. Soc. 2003, 125, 14272. (f)

Madrahimov, S. T.; Markovic, D.; Hartwig, J. F. J. Am. Chem. Soc. 2009, 131, 7228 .

[22] Leitner, A.; Shekhar, S.; Pouy, M. J.; Hartwig, J. F. J. Am. Chem. Soc. 2005, 127, 15506.

(Cheng, B.) 